\title{
Escenarios online para el aprendizaje de un instrumento musical: sinopsis de algunas investigaciones
}

\author{
Antonio Ángel Guillén Box ${ }^{1}$ \\ Conservatorio Profesional de Música de San Vicente del Raspeig \\ Rosabel Roig-Vila ${ }^{2}$ \\ Universidad de Alicante
}

Resumen: En este artículo realizamos una breve sinopsis de las conclusiones de algunas investigaciones en las que se ha experimentado el proceso de enseñanza-aprendizaje de un instrumento musical en escenarios online, centrándonos en el uso de la videoconferencia. Estas investigaciones realizadas en Canadá y Estados Unidos, han experimentado con diferentes instrumentos como el piano, el saxofón, la trompeta, el trombón y la tuba. La mayoría de las investigaciones en las que la experiencia de aprendizaje ha tenido lugar parcialmente o totalmente en un escenario online, coinciden en que para que el proceso de enseñanza-aprendizaje por videoconferencia sea óptimo, es necesario un cambio de metodología, una mayor preparación de las clases y la superación de las deficiencias de la tecnología en cuanto al proceso de transmisión de datos que ocasiona interrupciones en la comunicación. Se trata de un campo que ofrece multitud de posibilidades, pero también tiene sus limitaciones frente al contacto directo de la clase presencial en el aula física. Aspectos como la comunicación profesor-alumno, estrategias didácticas y determinados contenidos de la enseñanza musical, precisan de una atención diferente en un escenario online. En general, proponen el uso de la videoconferencia como complemento a contenidos asíncronos y a la clase presencial.

Palabras clave: E-learning, Videoconferencia, TIC, Internet.

Abstract: This article describes briefly the conclusions of the research project in which the process of teaching-learning of a musical instrument has been experimented in an online scenario, focusing on the use of video-conference. All the research activities have been implemented in Canada and the United States of America, using different instruments like the piano, saxophone, trumpet, trombone and tuba. Most of the research activities in which the learning experience has taken place partially or fully online coincide in that in order to the process of teaching-learning via video-conference to be successful, it is necessary the employment of a specific methodology, a better preparation of the lessons and the improve of the technology deficiencies regarding the data transmission process, that brings out interruptions in the communication. This process refers to a field that can offer multiple possibilities but also has some limitations against the direct contact that offers in-person lessons in a physical classroom. Different aspects like teacher-student communication, didactic strategies and specific music teaching content require a different attention in an online scenario. In general, they propose the use of video-conference as a complement to the asynchronous contents and to the in-person lesson.

Keywords: E-learning, Video-conference, ICT, Internet.

\section{Introducción}

\footnotetext{
1 Conservatorio Profesional de Música de San Vicente del Raspeig. E-mail: pedagogiamusical@hotmail.com Doctor en Investigación Educativa, Desarrollo Curricular y Profesional. Profesor de piano del Conservatorio Profesional de Música de San Vicente del Raspeig.

Titulado superior en las especialidades de Pedagogía Musical, Lenguaje Musical y Música de Cámara.

${ }^{2}$ Universidad de Alicante. E-mail: rosabel.roig@ua.es Doctora en Pedagogía, Directora del ICE de la Universidad de Alicante, directora de NAER-Journal of New Approaches in Educational Research,http://naerjournal.ua.es, directora del Grupo de Investigación EDUTIC-ADEI (VIGROB-0039), Profesora Titular de Universidad, Dpto. de Didáctica General y Didácticas Específicas, Universidad de Alicante
} 
El aprendizaje de las artes escénicas se ha desenvuelto comúnmente en escenarios en los que profesor y alumno compartían el mismo espacio y tiempo. O si realizamos una mirada más lejana en el tiempo, donde maestro y discípulo convivían durante un gran número de horas bajo el mismo techo.

La videoconferencia para la enseñanza de instrumentos musicales comenzó a utilizarse a mediados de 1990 (WILSON, 2013). Desde entonces, la videoconferencia ha avanzado en tecnología y con ello en calidad de imagen y sonido hasta cotas muy altas. Una sala o aula virtual debe tratar de proporcionar unas prestaciones semejantes a las que podrían ofrecerse en un aula in situ.

El escenario de aprendizaje que plantean las investigaciones a las que vamos hacer aquí referencia, se sitúa dentro de una clase individual transmitida por videoconferencia. La incorporación de las TIC en el aprendizaje de un instrumento musical, es algo que se está produciendo lentamente, resultando así interesante la observación de estas investigaciones. En relación a esto podemos referenciar a Salinas (2005) que señala:

Describir escenarios de aprendizaje propiciados por las TIC nos ayudará en el diseño y creación de ambientes de aprendizaje adecuados a las nuevas coordenadas espacio-temporales, a los nuevos objetivos educativos, etc., de tal forma que podamos comprender cómo los cambios afectan a los estudiantes, profesores, centros y a la comunidad. (p.423)

\section{Sinopsis de las investigaciones}

Romero (2015) realiza una investigación en Canadá a través de la Universidad de Athabasca cuyo título es A grounded theory study for the conversion of applied piano course at the graduate level to online environments, y cuyo estudio se situó en la provincia de Alberta (Canadá), aporta interesantes reflexiones sobre el conjunto del fenómeno online y su aplicación a la enseñanza en instrumentos musicales. Se trata de una investigación que persigue la extracción de propuestas que puedan ser utilizadas para guiar la realización de una formación de postgrado para el piano de forma online. En esta investigación cualitativa bajo el enfoque de teoría fundamentada y llevada a cabo a través de entrevistas, se plantea qué elementos de la enseñanza del piano que se dan normalmente en el aula física de carácter tradicional en el nivel de postgrado deben incluirse en los entornos online, según la opinión de los profesores de la provincia de Alberta. Los profesores más jóvenes tuvieron una actitud más abierta ante la enseñanza online del piano que los de mayor edad, los cuales manifestaban la necesidad de contacto para expresar muchas veces aspectos que mediante palabras no pueden. Los resultados de esta investigación fueron la creación de un diseño de estrategias efectivas para el aprendizaje del piano, sirviendo también como muestra para futuras investigaciones. La autora de esta tesis plantea que existen tres grandes retos respecto a la enseñanza online en un master de piano:

- Cómo enseñar adecuadamente aspectos de interpretación y psicomotricidad de forma online.

- Qué formación debe adquirir el profesor de piano de máster para poder dar clases de piano online.

- $\quad$ Una escasez de literatura que explique cómo debe ser realmente estructurada y planteada una clase de máster de piano en el aula física. 
Romero (2015) en su investigación con profesores de piano que impartían clase mediante videoconferencia describe qué circunstancias se produjeron:

- Disminución en la relación profesor-alumno.

- Imposibilidad de hacer uso de demostraciones que requieren contacto físico entre alumno y profesor.

- Muchos de los profesores opinaron que la enseñanza online obstaculiza en gran medida la percepción de la calidad sonora y sus matices, e s decir, existe una limitación tecnológica en este sentido.

- La visualización que el profesor tiene del alumno mientras éste realiza una interpretación al piano se ve mermada.

- Necesidad de una tecnología y una comunicación de alta calidad.

Romero (2015) concluye en su investigación que es necesario un contacto en el aula para mostrar los aspectos técnicos del aprendizaje instrumental. Y afirma que la metodología denominada "modelo conservatorio" sigue siendo usada en la metodología online, y en su investigación propone la creación de estrategias efectivas para el aprendizaje instrumental online.

Otra investigación en la que se experimentaba un escenario online es la de Lockett (2010), en la que a través de blendend learning, en su tesis Student perceptions about the effectiveness and quality of online musical instrument instruction, trata de descubrir las percepciones que los estudiantes tuvieron sobre aspectos de eficacia y calidad de la enseñanza instrumental online. Los resultados y aspectos que destacamos en su tesis publicada en la Universidad Capella de Estados Unidos, y que estuvo ceñida a la enseñanza online del bajo eléctrico son los siguientes:

- No existe diferencia significativa respecto a la eficacia del aprendizaje y la calidad de la instrucción.

- En la clase tradicional el profesor representa un papel muy importante. En la enseñanza online el papel protagonista lo comparten la tecnología y las cuestiones de comunicación.

- Una de las ventajas del eLearning en el instrumento musical, es que permite a personas de muy diversas edades y niveles educativos, tener acceso a este tipo de enseñanza musical.

- Un punto importante para el éxito del aprendizaje es que los alumnos tengan las expectativas claras de lo que se van a encontrar en el aprendizaje online.

En un instrumento muy diferente al piano y al bajo eléctrico como es la trompeta, hemos encontrado una investigación en la que se utilizó la videoconferencia 
síncrona mediante Skype como medio de clase. Esta investigación fue realizada por Dammers (2009) y muestra las siguientes conclusiones:

- Se trata de un formato funcional a nivel básico, para proporcionar información útil y conseguir progresos en el alumno.

- Durante la sesiones de videoconferencia, se produjeron numerosas limitaciones debido a la limitación visual y al retardo de la imagen.

- Debido a las limitaciones visual y al retardo de la imagen, el autor afirma que es un medio de clase que puede usarse solamente como complemento y no como sustituto de la clase presencial, dadas las limitaciones tecnológicas en el momento de realizar la investigación, y dado que no es un formato equivalente a la enseñanza instrumental cara a cara.

- Se produce una debilitación de los lazos emocionales entre profesor y el alumno, a pesar de tener contacto visual a través de la videoconferencia.

- También manifiesta que necesitó una mayor planificación y preparación de sus clases. Fue también muy importante la estructura de las lecciones y el turno de preguntas.

Una investigación que le otorgamos interés es la de Wilson (2013) titulada Bridging the virtual gap in Internet based music instruction: a feasibility study in trombone performance education, en la que pone en marcha un proyecto de aprendizaje online para trombón, mediante elementos de comunicación síncrona y asíncrona, con el propósito de situarlo como complemento a las clases presenciales y no como sustituto de éstas. El resultado final fue que sí que es posible un aprendizaje del instrumento por videoconferencia, pero ha de estar acompañado de contenidos de forma asíncrona para que el alumno pueda trabajarlos en casa de forma previa.

Wilson (2013) afirma que el aprendizaje online necesita más que una simple adaptación de la metodología de aprendizaje de un instrumento musical, pues son necesarias grandes innovaciones metodológicas para que pueda resultar exitoso este tipo de aprendizaje virtual en instrumentos de música.

Las herramientas TIC que Wilson (2013) utiliza en su investigación son:

- Videoconferencia a través de Google Hangouts: su utilización era siempre tras el estudio de la unidad de contenido que tenía asignada. Las explicaciones eran solamente de técnica instrumental, pues todas las explicaciones teóricas estaban insertadas en el blog.

- Blog multimedia (http://onlinetromboneteacher.blogspot.com.es/) para insertar contenidos de:

- Vídeo y audio del blog: fue el recurso más utilizado. 
- Textos e imágenes: se limitaba a breves aclaraciones.

Los alumnos con los que se llevó a cabo el estudio pertenecían a tres niveles diferentes y algunas de las reflexiones que llegó Wilson (2013) tras su investigación son:

- La clase online precisa de más duración que la del aula física, debido a los fallos de comunicación de Internet, entre otros.

- La calidad de sonido se vio bastante afectada, debido a los problemas tecnológicos de latencia en la transmisión, que fueron constantes en todas las clases, repercutiendo también la diferencia de equipamiento y acústica entre el aula del profesor y la del alumno.

- Resultó dificultoso corregir aspectos interpretativos del instrumento mediante el uso de la videoconferencia, cuyo origen era la mala calidad de la imagen y el sonido. A pesar de ello, los alumnos sí que pudieron aprender gracias al apoyo de la parte de contenidos asíncronos que contenía el blog.

- El uso de vídeos en YouTube con demostraciones del profesor de cómo interpretar fragmentos, suplió en parte las deficiencias de la videoconferencia.

- La autoevaluación del alumno mediante el visionado de sus grabaciones, fue un recurso utilizado, el cual proporcionó gran interés educativo para fomentar la reflexión del alumno sobre sus propias interpretaciones.

- En el blog se mostraban los objetivos que tenía que perseguir el alumno para la próxima clase por videoconferencia. Esta planificación de contenidos facilitó el aprendizaje en gran medida, para que las clases por videoconferencia fuesen bien aprovechadas.

- Sí que es posible un aprendizaje del instrumento por videoconferencia, pero ha de estar acompañado de contenidos de forma asíncrona para que el alumno pueda trabajarlos en casa de forma previa, además de una adaptación en la metodología.

- Debido a los fallos de comunicación, la clase debe tener una mayor duración.

- La latencia es otro gran inconveniente afectando al desarrollo de la clase.

- La tecnología supone dificultad en gran medida el aprendizaje debido a la mala calidad de la imagen y el sonido.

- Como aspecto positivo señala la posibilidad de que la auto-grabación pueda utilizarse como recurso educativo.

Otra investigación que queremos referenciar es la realizada en Estados Unidos por Carter (2013), en la que pone en marcha un proceso de enseñanza online en 
estudiantes de edades entre seis y nueve años sin conocimientos previos de piano. El título de esta tesis es Musical achievement and attitutde of beginning piano students in a synchronous videoconferencing lesson environment, en la que se utilizó la videoconferencia (mediante Skype) que trataba de explorar los elementos utilizados en la instrucción, detectando el nivel final alcanzado por los estudiantes y su actitud hacia las clases. En esta investigación se reflexionó también hasta qué grado las clases a distancia pueden ser una alternativa viable para la enseñanza cara a cara o tradicional. El período de prueba fue de siete meses con una carga lectiva de media hora a la semana, durante el cual se estudió dos grupos de alumnos: un grupo al que se le impartía enseñanza tradicional en el aula física y otro grupo el cual recibía formación mediante enseñanza online. Para evaluar niveles de aprendizaje se tuvieron en cuenta aspectos como:

- Interpretación de una pieza musical que el alumno había estudiado.

- Un examen de lectura a primera vista, memoria auditiva y memoria visual.

- Entrevista final.

- Encuesta de actitud.

Tanto la clase en el aula física como la clase en el aula online, fueron grabadas en vídeo con objeto de observar en cada grupo qué elementos (explicaciones del profesor, preguntas del alumno, interpretación de las piezas musicales) se invertía el tiempo. Esta grabación se hizo en tres momentos diferentes del período observado en cada grupo: al inicio de su estudio, a mitad de su estudio y al final cuando la obra ya estaba terminada.

La investigación de Carter se llevó a cabo con pianos electrónicos mediante el uso del sistema MIDI para conectar los teclados del alumno y el profesor de forma simultánea. Gracias a esta interconexión, al presionar la tecla en uno de los pianos sonaba la misma tecla en el otro piano y se visualizaba la nota musical en la pantalla del teclado. Además disponía de un silenciador automático del micrófono para que, cuando se tocara en uno de los instrumentos, el sonido generado no produjese un acople a través del micrófono.

De la investigación de Carter (2013), extraemos una serie de conclusiones y descripciones que consideramos interesantes, pese a que el proceso de enseñanzaaprendizaje fue llevado a cabo con pianos electrónicos y no acústicos. Los aspectos que destacamos son en relación a la metodología de clase y cómo afectó la separación física entre profesor y alumno de piano:

- No se encontraron diferencias significativas en relación al medio empleado (aula física o aula online) en cuestión de rendimiento.

- Los alumnos del aula física obtuvieron una calificación ligeramente más alta que el grupo de alumnos que recibió clases online en las cuatro tareas de rendimiento propuestas, pero la diferencia no era notable para considerarse importante. 
- Los alumnos de la clase online, en una primera etapa, emplearon mucho más tiempo que el grupo del aula física en resolver aspectos de dudas y problemas surgidos con la tecnología que estaban usando.

- El tiempo destinado en la clase online para realizar juegos interactivos (tocar ambos con un ritmo de palmas, tocar a dúo con los teclados, etc.) fue muy superior al destinado en el aula física, además de que algunos ejercicios interactivos no fueron del todo posibles realizarlos online.

- Las estrategias didácticas para una clase de piano online deben ser otras a las de la clase tradicional.

- El concepto, para estos niveles iniciales, que costó más enseñar mediante la clase online fue el ritmo, pues precisa de una simultaneidad y una exactitud en la realización que impedían el retardo producido por las deficiencias de conexión de Internet. Esta dificultad se trató de subsanar evitando la realización síncrona de estos ejercicios rítmicos, pasándolo a realizar en un orden consecutivo: el profesor propone el ritmo modelo y el alumno lo imita después.

- La interacción simultánea multitarea que suelen realizar los profesores de piano al corregir al alumno mientas interpretan se vio afectada de forma que no era posible: cada acción del profesor (explicación) debía procesarse por separado, permaneciendo el alumno en silencio y sin tocar el instrumento.

- En la enseñanza online, el estudiante asume un número mayor de responsabilidades, pues ha de estar pendiente de más componentes.

- Dado que en la enseñanza online no es posible el contacto físico para reconducir posturas erróneas del alumno, es posible que los alumnos, cuyo aprendizaje es mayoritariamente kinestésico, se vean perjudicados en este sentido.

- Los profesores online han de usar descripciones muy precisas en sus métodos de enseñanza y tener facilidad oratoria para poder explicar aquellos aspectos reconducción postural.

- Es necesario crear una rutina de trabajo para aprovechar al máximo el tiempo en las clases online.

- Uno de los motivos que preocupan y que pueden causar dudas en cuanto a la fiabilidad de la educación online como alternativa al método tradicional, son la calidad de transmisión, la fiabilidad y la ausencia de la relación personal entre profesor y alumno. 
- Los avances tecnológicos han ido por delante de la didáctica eLearning del piano, siendo la enseñanza privada no académica, la única depositaria de experiencias eLearning en este campo. Carter afirma que existen carencias en la enseñanza online del piano debido a su corta experiencia, siendo necesario una determinación concreta de qué estrategias didácticas han de usarse para este ámbito.

- Respecto a la metodología es necesario utilizar otras estrategias a las de la clase tradicional, pues entre otros factores han de usar en el aula instrucciones muy precisas y tener una facilidad oratoria para explicar aspectos de reconducción postural.

Orman y Whitaker (2010) llevaron a cabo un estudio para observar el rendimiento de alumnos de saxofón y tuba a través de un aprendizaje online combinado con aprendizaje presencial, obteniendo como resultado los siguientes aspectos:

- Aumento del contacto visual alumno-profesor, comprobación que se hizo analizando los fotogramas de las sesiones grabadas en vídeo.

- Disminución del tiempo dedicado por el profesor a mostrar ejemplos tocando las piezas (aprendizaje por modelado), lo que supuso un aumento del tiempo en que los alumnos tocaban las piezas.

- Disminución en el número de comentarios ajenos a la clase (sobre ocio o bromas), disminuyendo así el número de distracciones.

- $\quad$ Aparición de problemas tecnológicos de comunicación.

Las conclusiones de Orman y Whitaker (2010) fueron que, a pesar de generar elementos positivos en este tipo de aprendizaje, en el momento de hacer el estudio todavía la tecnología no estaba a la altura de proporcionar una clase online de calidad como para reemplazar a una clase presencial, aunque sí le veían ventajas para el aprendizaje en zonas en las que por su circunstancia geográfica no existían centros de enseñanza musical. Consideraban que el aprendizaje online puede tener un carácter funcional y tal vez más eficiente en algunos aspectos. Sin embargo, los problemas de compresión, y sobre todo la calidad de audio, impedían el desarrollo de todo su potencial.

\section{Conclusiones}

En el conjunto de las investigaciones aquí expuestas se aprecia en general la coincidencia de que en el desarrollo de una clase de instrumento musical por videoconferencia se producen circunstancias que hacen que no pueda seguirse el mismo diseño que el de una clase presencial en el aula física. En general, cualquier asignatura impartida por videoconferencia implica cambios y diferencias respecto al 
aula tradicional, pero en el caso de la clase de instrumento musical, quizás aún más como pueden ser:

- Cambios en el proceso de comunicación profesor-alumno.

- Cambios en la metodología.

- Variación de la distribución del tiempo de aula.

- Dificultades en el proceso de enseñanza-aprendizaje de ciertos contenidos musicales y de técnica del instrumento.

- Problemas derivados de la tecnología en si como soporte que dificulta la comunicación y la transmisión de sonido del instrumento musical.

Por ello se hacen necesarias continuas y nuevas experimentaciones que pongan en marcha nuevos mecanismos y estrategias dentro del proceso de enseñanzaaprendizaje del instrumento musical, que signifiquen una resolución a estas dificultades y una adecuación a los cambios que conlleva el desarrollo de una clase por videoconferencia.

Para la enseñanza de un instrumento musical, estamos en la fase inicial de experimentación, descubrimiento y desarrollo de este nuevo escenario online, hasta poder llegar en un futuro a una valoración y uso más óptimo de estos mecanismos.

\section{Referencias}

Carter, R. (2013). Musical achievement and attitude of beginning piano students in a synchronous videoconferencing lesson environment. A Dissertation Submitted to the Graduate Faculty of the Louisiana State University and Agricultural and Mechanical College in partial fulfillment of the requirements for the degree of Doctor in Philosophy. Lousiana State University.

Dammers, R. J. (2009). Utilizing Internet-based videoconferencing for instrumental music lessons. Update: Applications of Research in Music Education, 28 (1), $17-24$.

Orman, E. K. and Whitaker, J. A. (2010). Time usage during face-to-face and synchronous distance music lessons. American Journal of Distance Education, 24 (2), 92-103.

Romero, A.M. (2015). A grounded theory study for the conversion of applied piano course at the graduate level to online environments. A thesis submitted to the faculty of graduate studies in partial fulfillment of the requirements for the degree of master of education in distance education (MED). Athabasca University.

Salinas, J. (2005). Nuevos escenarios de aprendizaje. Grupo CIFO: IV Congreso de Formación para el Trabajo. pp. 421-431. IFES, Fundación Forcem y Universidad de Vigo. 
Wilson, A.J (2013). Bridging the virtual gap in Internet based music instruction: a feasibility study in trombone performance education. Doctoral thesis. University of North Carolina at Greensboro.

Recebido para publicação em 28-08-16; aceito em 23-09-16 